\title{
NOA - Von Demonstratoren zu Pilotanwendungen
}

Vier Anwendungsfälle der Namur Open Architecture

\begin{abstract}
Das Konzept Namur Open Architecture (NOA) wurde auf der Namur-Hauptsitzung 2016 vorgestellt, im Jahr 2017 gab es erste Demonstratoren. Inzwischen werden drei konkrete Elemente des NOA-Konzeptes erarbeitet: Die NOA-Diode sorgt für den sicheren Datenexport aus der Namur-Pyramide, das NOA-Informationsmodell beschreibt die Daten und Bezeichnungen, und Verification of Request erlaubt einen kontrollierten Datenfluss zurück in das Prozessleitsystem der Anlage. Diese Elemente wurden an der IGR-Testanlage im Industriepark Höchst implementiert. Im Beitrag werden vier konkrete Anwendungsfälle für die NOA-Bausteine vorgestellt: eine Pumpendiagnose, die automatische Aktualisierung der Dokumentation nach einem Sensortausch, die Online-Überwachung eines Prozessanalysegerätes sowie ein Dimensionierungs-Check für Feldgeräte.
\end{abstract}

\section{SCHLAGWÖRTER NOA / Namur Open Architecture / NOA-Diode /}

NOA-Informationsmodell / Verification of Request / Anwendungsbeispiel

\section{NOA - from demonstrators to pilot applications - Four applications of Namur Open Architecture}

The Namur Open Architecture (NOA) concept was presented at the Namur Annual General Meeting in 2016 and the first demonstrators were shown in 2017. Three building blocks of the NOA concept are now being developed: The NOA Diode ensures the secure export of data from the Namur pyramid, the NOA Information model describes the data and nomenclature, and Verification of Request allows a controlled data flow back into the control system of the plant. These elements were implemented at the IGR demonstration plant at the Höchst Industrial Park. This paper presents four monitoring + optimization $(\mathrm{M}+\mathrm{O})$ applications for the NOA concept: pump monitoring, automatic updating of documentation after sensor replacement, online monitoring of a process analyser, and a dimensioning check for field devices.

KEYWORDS NOA / Namur Open Architecture / NOA diode / NOA information model / verification of request / use cases 


\author{
JAN DE CAIGNY, BASF \\ THOMAS TAUCHNITZ, Tautomation.consulting \\ RONNY BECKER, Bilfinger \\ CHRISTIAN DIEDRICH, TIZIAN SCHRÖDER, Otto-Von-Guericke-Universität \\ DANIEL GROSSMANN, SUPRATEEK BANERJEE, TH Ingolstadt \\ MARKUS GRAUBE, LEON URBAS, TU Dresden
}

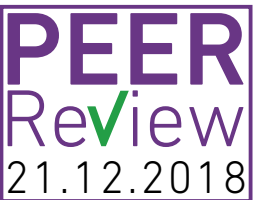

ahr für Jahr hat das Namur-Open-ArchitectureKonzept (NOA) weitere Schritte in Richtung industriereifer Lösungen gemacht. Nachdem auf der Namur-Hauptsitzung im Jahr 2017 individuelle Demonstratoren gezeigt wurden, ist im Jahr 2018 die erste Industrie-4.0-Testanlage im Bereich der Prozessindustrie in Betrieb genommen worden. Während der Namur-Hauptsitzung 2018 wurden sowohl diese Anlage als auch verschiedene implementierte NOA Monitoring + Optimization (M+O) Use Cases vorgestellt und konnten über eine Live-Verbindung mit der Anlage aus der Ferne verfolgt werden - Industrie 4.0 live in action auf der Namur-Hauptsitzung!

In diesem Beitrag wird die IGR-Testanlage vorgestellt, um dann die vier neuen NOA M+O Use Cases detailliert vorzustellen:

- einen Pumpenüberwachungsdienst,

- einen automatisch erzeugten As-built-Stand in einem Planungstool nach einem Feldgerätetausch,

- einen M+O-Dienst für ein typisches Prozess-AnalyseGerät und

- einen automatisierten Dimensionierungscheck für alle eingebauten Feldgeräte.

- Für ein besseres Verständnis der umgesetzten Use Cases, werden vorab die sogenannten NOA-Bausteine vorgestellt, die bei den implementierten Demonstratoren eine wichtige Rolle spielen.

\section{BAUSTEINE DER NOA-ARCHITEKTUR}

Die NOA-Architektur [1, 2, 3]) besteht aus drei Hauptbausteinen: NOA-Informationsmodell (IM), NOA-Diode (Security) und Verification of Request (siehe Bild 1). Über das NOA-Informationsmodell werden Gerätedaten auf einheitliche Art und Weise über einen OPC-UA-Server (den sogenannten NOA-Server) zur Verfügung gestellt. Dabei spielt es keine Rolle, ob es sich um klassische PLTGeräte handelt oder aber um $\mathrm{M}+\mathrm{O}-$ Sensoren, die nicht der Prozessführung dienen und dementsprechend nicht Bestandteil der Core Process Control (der Kernautomatisierung, mit der die Produktionsanlage gesteuert, geregelt und überwacht wird) sind. Das NOA-Informationsmodell kann dabei im Gerät selbst implementiert sein oder aber über weitere Systemkomponenten oder Tools repräsentiert werden (beispielsweise für Bestandsanlagen mit aktuellen Feldgeräten). Die Inhalte des Informationsmodells richten

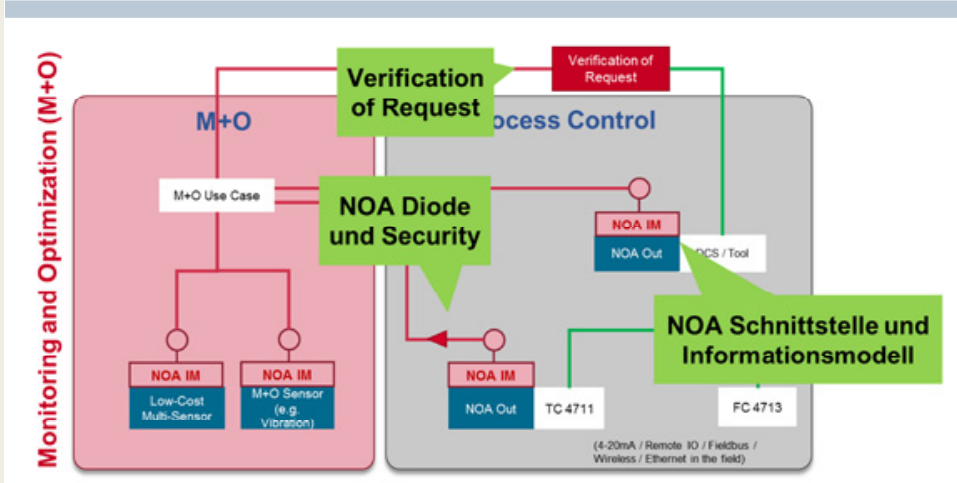

BILD 1: NOA Bausteine
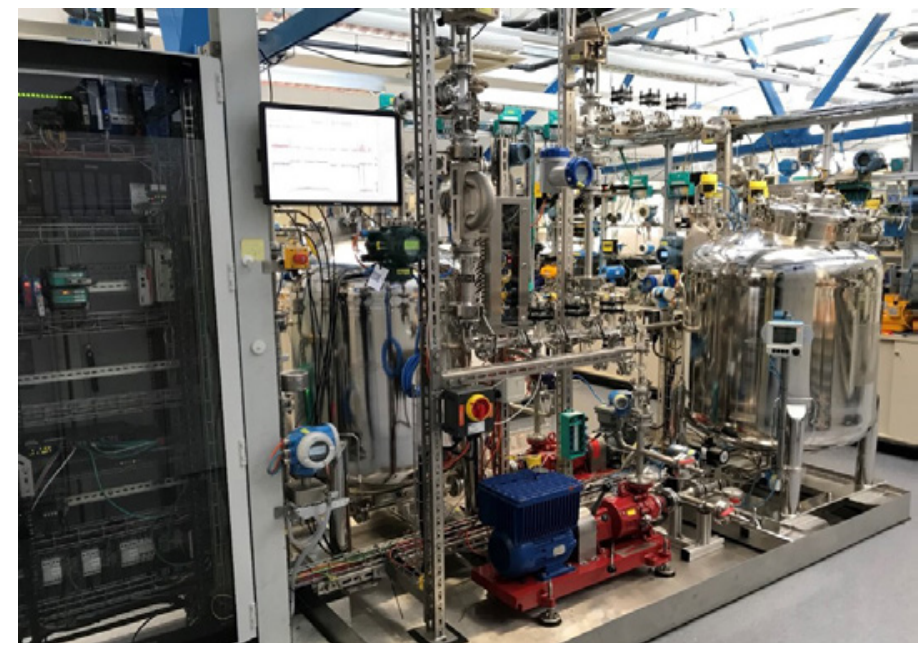

BILD 2: Industrie-4.0-Testanlage der IGR bei Bilfinger Maintenance $\mathrm{GmbH}$ 


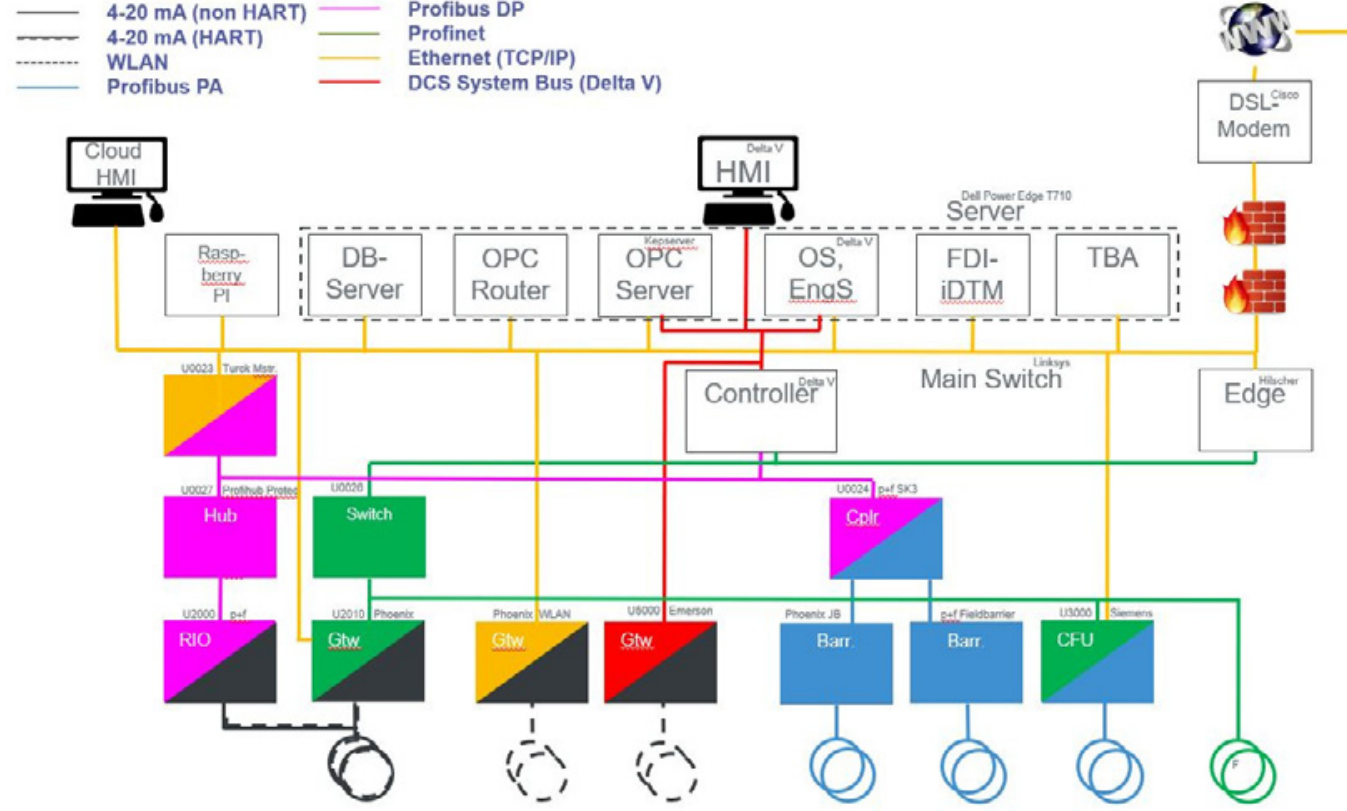

BILD 3: Systemstruktur der Testanlage

sich nach den Parametern, die im NOA-Arbeitskreis erarbeitet wurden und die in der entsprechenden NamurEmpfehlung spezifiziert werden. Ein erster Entwurf des NOA-Informationsmodells, der industrieweit in Abstimmung ist, liegt bereits vor und wird bei den nachfolgend erläuterten Demonstratoren verwendet.

Für die Umsetzung der NOA-Use Cases werden die Daten über das NOA-Informationsmodell ausgelesen und beispielsweise in einer Cloud-Applikation verarbeitet. Die NOA-Diode dient dazu, die Rückwirkungsfreiheit auf das Prozessleitsystem sicherzustellen, sodass die Verfügbarkeit der Anlage gewahrt bleibt. Wenn durch eine M+O-Applikation Erkenntnisse entstehen, die zu einer Änderung an der Anlage führen (beispielsweise neue Reglerparameter bei einem oszillierenden Regelkreis), so wird diese Anfrage (Request) durch die verantwortlichen Personen verifiziert und für das Prozessleitsystem freigegeben (Verification of Request, [7]). Diese Bausteine lassen sich flexibel kombinieren, sodass NOA für verschiedene Anlagenszenarien einsetzbar ist. Diese decken Bestandsanlagen mit bestehenden Feldgeräten, Anlagen mit Ethernet-basierten Feldgeräten, Szenarien für den reinen (gegebenenfalls temporären) Einsatz von $\mathrm{M}+\mathrm{O}$ Sensoren sowie eine Kombination dieser Szenarien ab.

\section{IGR-TESTANLAGE FÜR INDUSTRIE 4.0}

Das Interesse an Industrie 4.0 ist groß. Es gibt viele Produkte und Konzepte - da ist es nur konsequent, eine prozesstechnische Anlage zu haben, in der neue Produkte und Konzepte ohne Risiken hinsichtlich der Prozesse und der IT Security realistisch getestet werden können. Mit diesem Ziel hat die Bilfinger Maintenance im Auftrag der Interessengemeinschaft Regelwerke Technik (IGR) e.V. im Industriepark Frankfurt-Höchst eine Testanlage errichtet. Diese Anlage wurde für die Use Cases der NOADemonstratoren bei der Namur-Hauptsitzung 2018 in Bad Neuenahr verwendet. Im Vergleich zu den Use Cases des Vorjahres, die auf separaten Testumgebungen implementiert wurden, gab dies in Verbindung mit dem RemoteZugriff direkt aus Bad Neuenahr einen ersten Eindruck, wie NOA in der betrieblichen Praxis funktionieren kann.

Die Hauptapparate der IGR-Testanlage sind zwei Behälter und zwei modifizierte Pumpen, mit denen ungefährliche Flüssigkeiten durch Edelstahl- und Glasleitungen in verschiedenen Kreisläufen gepumpt werden können, siehe Bild 2. So einfach dieser verfahrenstechnische Teil ist, so realitätsnah und umfangreich ist die mit zirka $200 \mathrm{E} / \mathrm{A}$ Punkten umgesetzte Prozessleittechnik, deren Struktur in Bild 3 dargestellt ist. Diese beinhaltet unter anderem:

- Feldgeräte-Anschluss

- mit 4-20 mA: ohne und mit HART-Signal,

- mit Wireless HART,

- mit Profibus PA, Profibus DP, Profinet,

- Remote I/Os und Wireless-HART-Sender/Gateways,

- Umsetzer von 4-20 mA (ohne und mit HART-Signal) auf Profibus DP, Profinet und TCP/IP,

- Umsetzer von Profibus PA auf Profibus DP und Profinet

- Umsetzer von Profibus DP auf TCP/IP,

a Edge-Gateway von Profinet auf TCP/IP, 


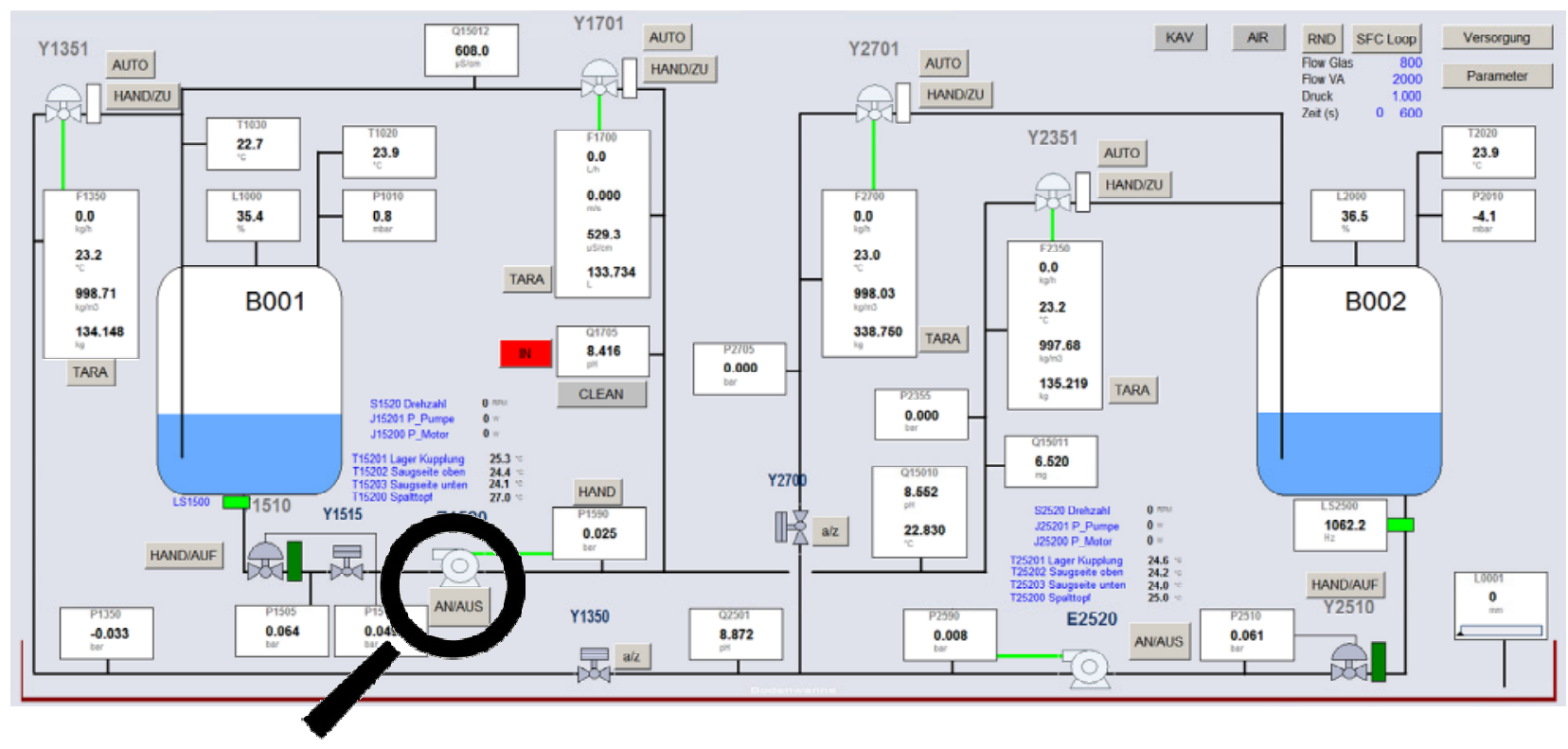

BILD 4: Pumpe des Use Cases im R\&I-Fließbild

- Prozessleitsystem mit Controller, Operator Station, Engineering Station und OPC-Server,

- Server mit Datenbanken, OPC-Router, FDI-iDTM,

- Anschluss an die Cloud via Firewalls und DSL-Modem. Soweit möglich, wurden vorhandene Standards genutzt und herstellerspezifische Lösungen vermieden. Die IGRTestanlage ist seit Anfang 2017 mit der Microsoft Azure Cloud verbunden. Inzwischen wurden mehr als 4 GB Prozessdaten gespeichert und stehen für Auswertungen zur Verfügung.

Die IGR-Testanlage ist besonders dafür geeignet, typische Aufgabenstellungen und Arbeitsabläufe der Prozessindustrie zu testen. Fehlerzustände wie Gasblasen oder Alterungseffekte können definiert eingestellt werden, um aussagefähige Daten zu erhalten und beispielsweise Diagnosesoftware zu testen. Diese Möglichkeiten sind in den auf der Namur-Hauptsitzung 2018 gezeigten Use Cases umfänglich einbezogen worden.

Neue Entwicklungen wie Field Device Integration (FDI), Advanced Physical Layer (APL), Modular Type Package (MTP, [4]) und NOA können so unter praxisnahen Randbedingungen gründlich getestet werden. Ein Einsatz neuer Technologien in der Praxis kann durch Schulungen vorbereitet und unterstützt werden. Die Möglichkeiten dieser Anlage werden bereits durch zahlreiche Anlagenbetreiber, Hochschulen, Organisationen und mehr als 10 Hersteller von Automatisierungsgeräten und -systemen genutzt. Durch die gute Zugänglichkeit und Ungefährlichkeit der Anlage können neue Versuche schnell und unkompliziert durchgeführt werden.

\section{NOA USE CASE 1: PUMPENDIAGNOSE \\ 3.1.Aufgabenstellung}

Der Demonstrator der Otto-von-Guericke-Universität/ ifak Magdeburg ist der Pumpendiagnose gewidmet. Die Zielsetzung ist es, typische pumpenbezogene oder prozessbezogene Fehlerfälle oder Anomalien zu erkennen. Dazu zählen beispielsweise Kavitation, Bläschenbildung im Medium oder Schäden an der Lagerschale.

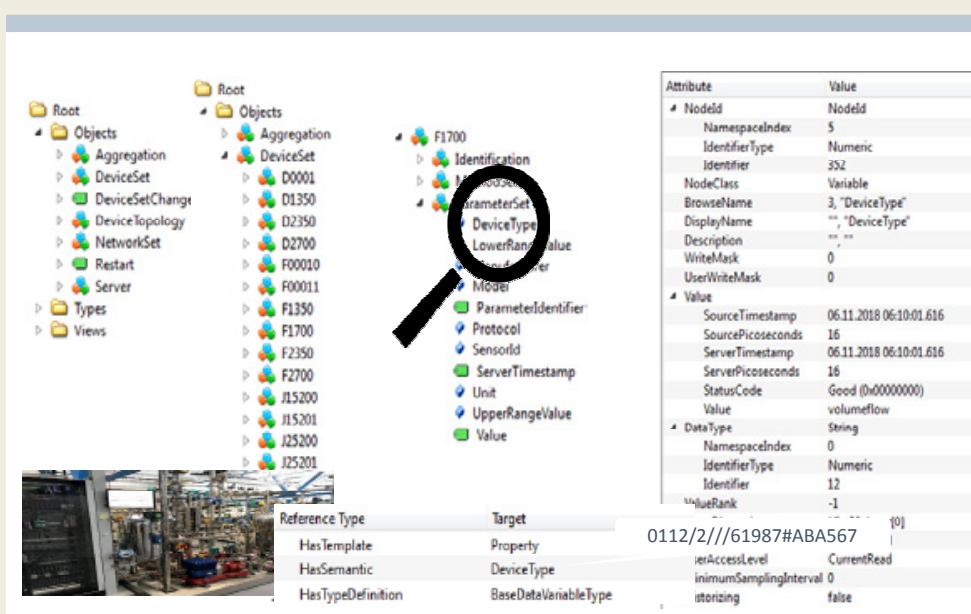

BILD 5: Informationsmodell im NOA-Aggregated OPC-UA-Server 


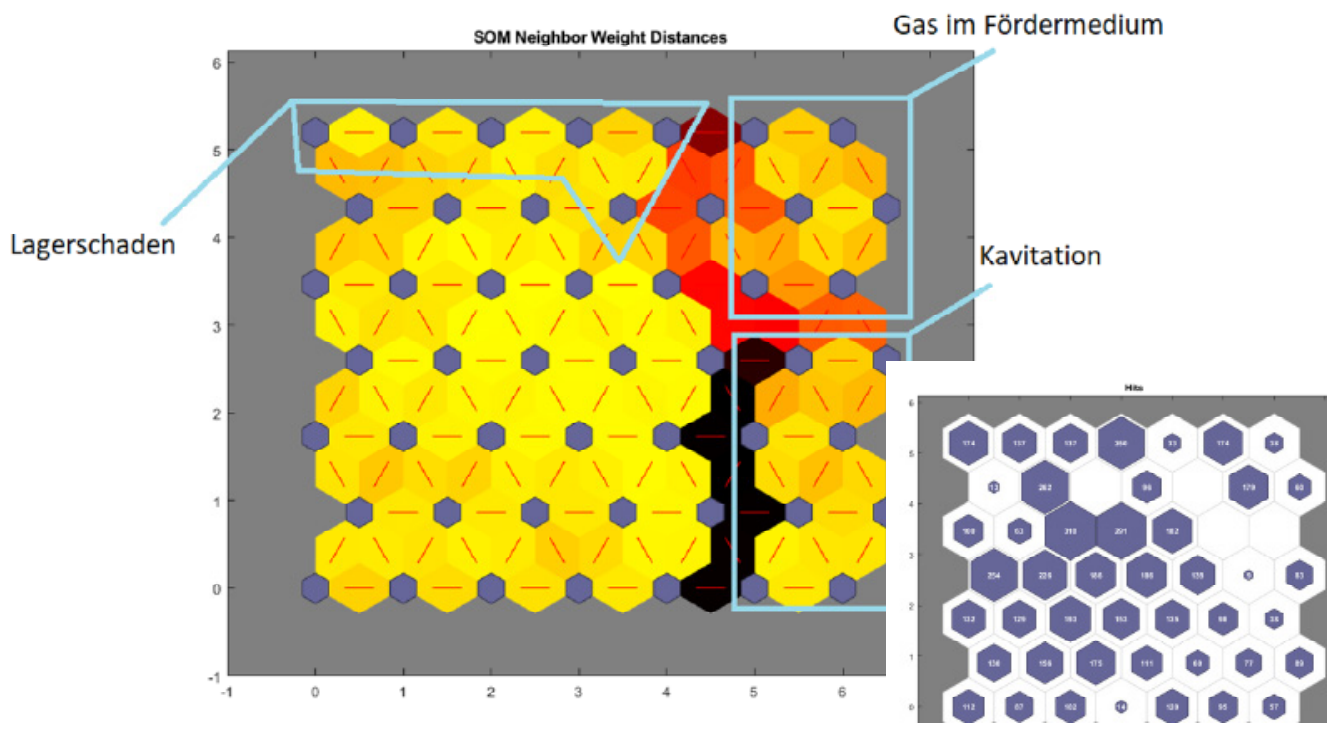

BILD 6: Angelernte Selbstorganisierte Karte (Self Organizing Map, SOM) der Pumpenüberwachung mit Fehlerund Anomaliefällen

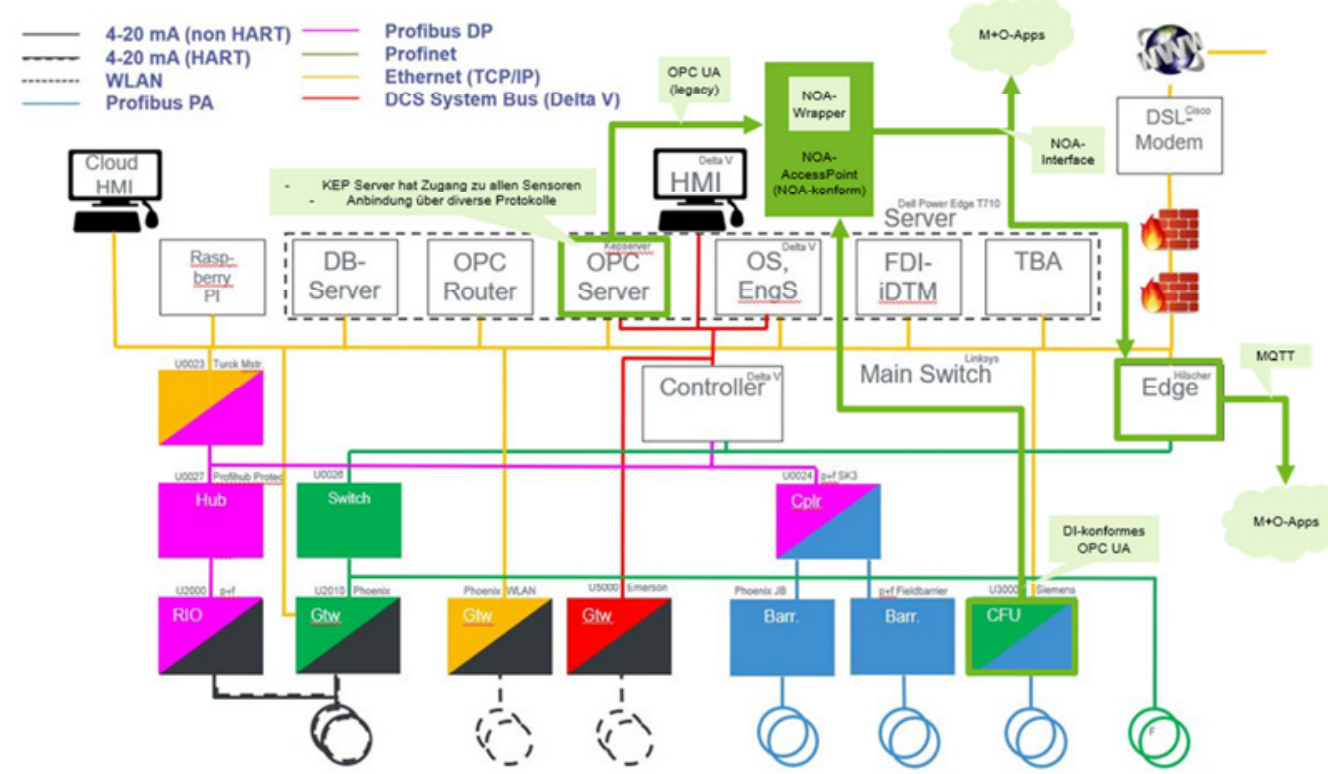

BILD 7: Datenfluss in der IGR-Anlage (grün hervorgehoben)

Der Use Case zeigt, wie das NOA-Konzept, ohne Änderungen im Engineering, in der Automatisierungslösung des Leitsystems rückwirkungsfrei umgesetzt werden kann. Dazu werden Prozessgrößen, die sich bereits in der Anlage befinden, derart miteinander verknüpft, dass eine Identifikation der besagten Fälle möglich ist [5].

\subsection{Aufbau des Demonstrators}

Die Pumpe bewirkt den Transport des Wassers zwischen den beiden Behältern der IGR-Testanlage (Bild 4). Die Instrumentierung misst den Druck, den Durchfluss und die Temperatur, sowohl vor als nach der Pumpe. Diese Prozessgrößen können für die Diagnose verwendet werden. Es wird dabei auf zusätzliche 

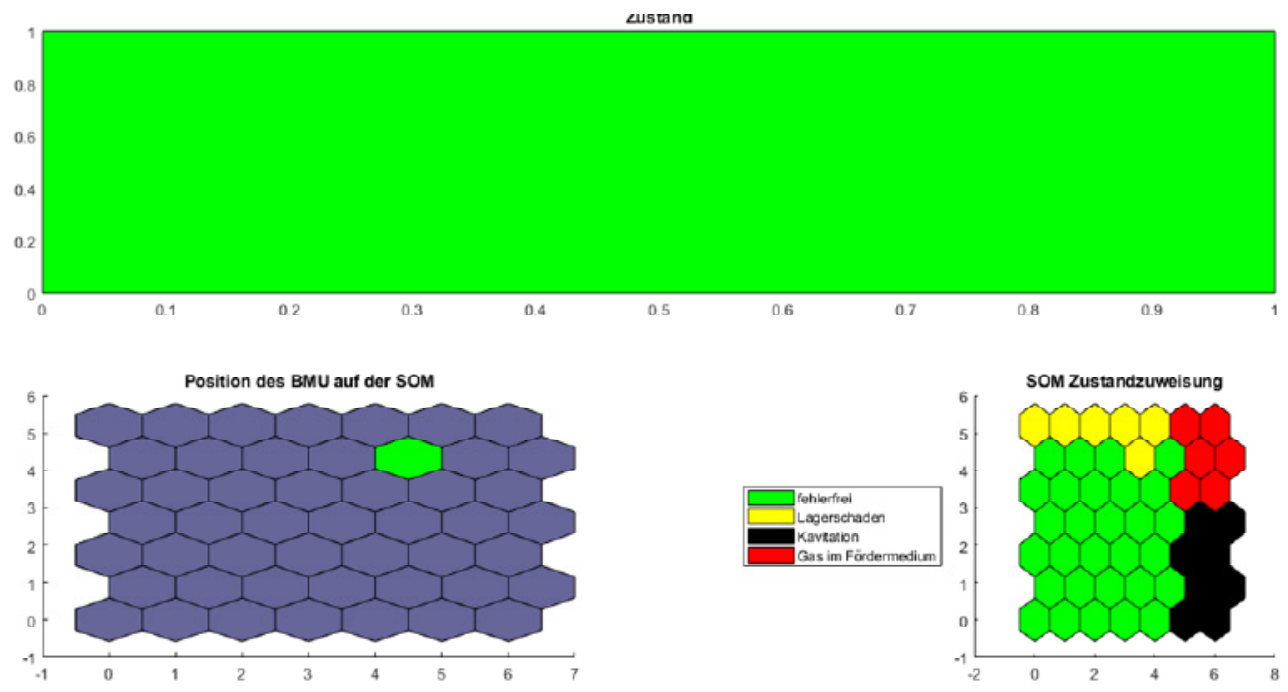

BILD 8: GUI der Pumpenüberwachung auf Basis der SOM

Sensorik, beispielsweise auf einen Vibrationssensor an der Pumpe, verzichtet, um zu evaluieren, ob nutzbare Informationen auch ohne diesen zusätzlich zu installierenden Sensor zu gewinnen sind.

Die Umsetzung des Datenzugangs im Use Case erfolgt mit dem NOA-Informationsmodell im NOA-Aggregated OPC-UA-Server der IGR-Testanlage (Bild 5). Von links nach rechts sind zu erkennen: Die Root-Ebene des Informationsmodells, in dem sich die Geräteliste (DeviceSet) befindet, die in der zweiten Spalte detailliert zu erkennen ist. In der dritten Spalte ist beispielhaft ein Durchflussmessumformer (F1700) ausgewählt, bei dem bereits die ihm eigene Parameterliste aufgeklappt wurde. Jeder Parameter hat eine standardmäßig definierte Attributliste, in der beispielsweise der Datentyp und der Name enthalten sind (rechte Spalte). Außerdem besitzt jeder NOA-Parameter eine semantische Referenz (HasSemantic), die auf den in der IEC 61987 definierten International Registration Data Indentifier (IRDI) verweist. Die Anwendungen der Use Cases kennen diesen IRDI und können über Standardmechanismen von OPC UA danach suchen. Dies muss zunächst mit allen für den Use Case vorgesehenen Parametern erfolgen. Im Fall der Pumpenüberwachung sind dies die erwähnten Durchfluss-, Druck- und Temperaturprozessgrößen.

Zur Fehler- und Anomalieerkennung werden sogenannte selbstorganisierende Karten (Self Organizing Map, SOM), eine Art von künstlichen neuronalen Netzen für unüberwachtes Lernverfahren, verwendet. Ziel ist es, die beschriebenen Fehlerfälle im laufenden Betrieb zu erkennen. Dazu wurde die SOM mit fehlerfreien und fehlerbehafteten Zuständen angelernt (Bild 6). Die Fehlerzustände wurden manuell im laufenden Betrieb durch die
IGR-Mitarbeiter eingebracht. Fünf separate Datensätze (je mit fehlerfreiem und fehlerbehaftetem Zustand) wurden zu einem Datensatz für das Lernen zusammengefügt. Insgesamt standen knapp 5900 Datenvektoren mit sechs Prozessgrößen zur Verfügung. Diese wurden zunächst normalisiert. Ab einer SOM-Größe von 7x7 konnten reproduzierbare Ergebnisse erzielt werden. Größere Matrizen brachten keine zusätzlichen Informationen. Die Matrix in Bild 6 (rechts unten) zeigt die Häufigkeit der Gewinnerneuronen beim Anlernen.

\subsection{Ergebnisse}

Die Kommunikationsverbindung zu den Geräten der Anlage erfolgt über verschiedene Pfade (Bild 7). Einige Prozessgrößenzugänge nutzen OPC-Schnittstellen, die durch eine spezielle OPC-Server-Komponente und einen entsprechenden NOA-Wrapper in den NOAAggregated OPC-UA-Server umgesetzt werden. Der Wrapper übernimmt hierbei die Umwandlung der proprietären OPC-Formate auf NOA-konformes OPC UA. Die weiteren Prozessgrößen werden über einen

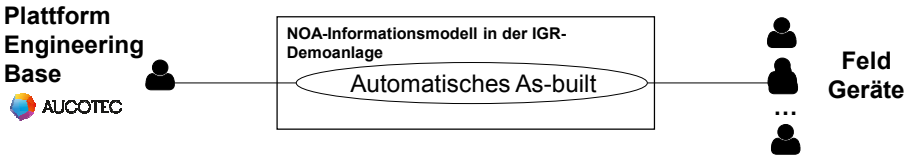

BILD 9: Komponenten des Use Cases Automatisierte Aktualisierung der Anlagendokumentation 

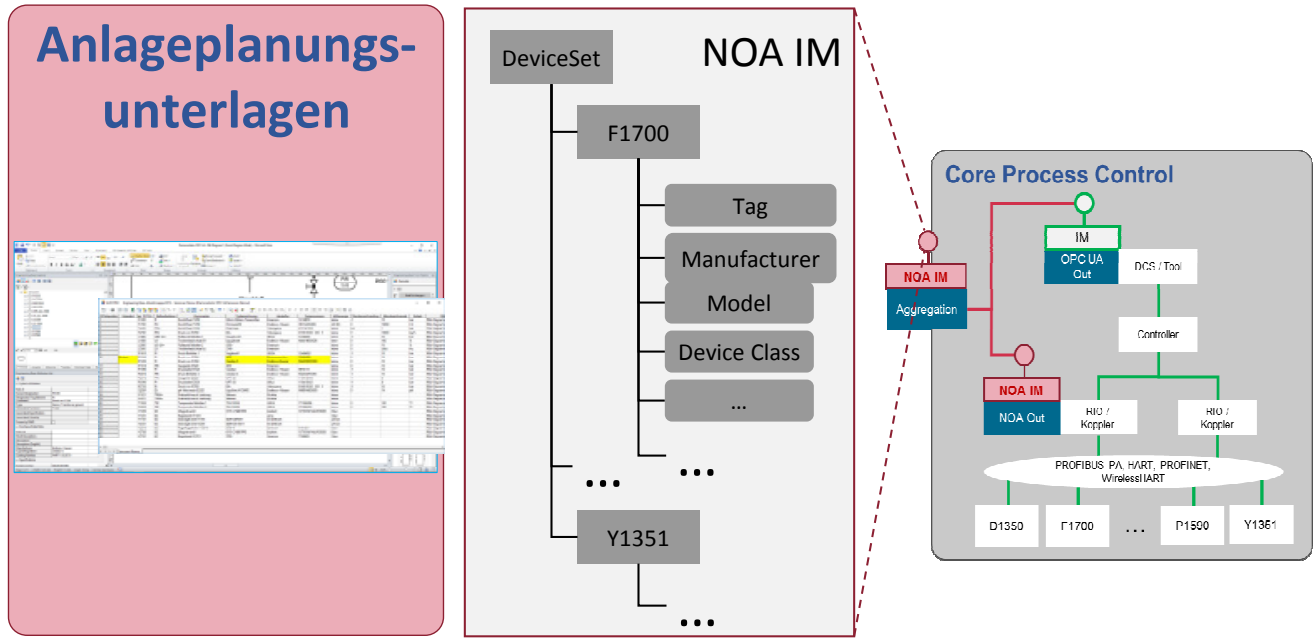

BILD 10: Einordnung des Use Cases in die NOA-Architektur

Remote I/O, der schon mit einem NOA-konformen OPCUA-Server ausgestattet ist, ohne zusätzliches Wrapping direkt in den Aggregated Server eingespielt. Der NOA-Aggregated OPC-UA-Server dient dabei dann als einheitlicher Zugangspunkt zu den benötigten Prozessdaten für die Anwendung. Die Umsetzung des SOM erfolgt mittels Matlab, dessen existierende Bibliotheken verwendet werden.

Mit einer für diese Anwendung passende Aktualisierungsrate von 1-2 s (eingestellt im NOA-Aggregated OPC-UA Server) wurden die Datenvektoren in das angelernte SOM eingespielt. Die Nutzerschnittstelle der Anwendung ist in Bild 8 dargestellt. Der oben dargestellte horizontale Balken zeigt den Zustand der Anlage: Grün entspricht einem fehlerfreien Zustand, gelb signalisiert einen Lagerschaden, schwarz signalisiert Kavitation und rot steht für Bläschenbildung. Links unten ist das Neuron farblich hervorgehoben, auf dem der aktuelle Pumpenzustand gemappt worden ist. Während der Namur-Live-Demonstration wurden die drei Fehlerzustände manuell in die Anlage eingebracht. Der entsprechende Farbumschlag konnte unmittelbar in Bad-Neuenahr beobachtet werden.

\section{NOA USE CASE 2: AUTOMATISIERTE} AKTUALISIERUNG DER ANLAGENDOKUMENTATION 4.1 Aufgabenstellung

Der Anwendungsfall Automatisierte Aktualisierung der Anlagendokumentation (OvGU/ifak Magdeburg zusammen mit Aucotec Hannover) veranschaulicht, wie die Anlageplanungsunterlagen in einem Engineering-System während des operativen Betriebes ständig aktuell gehalten werden können. Dies wird erreicht, indem mindestens ein OPC-UA-Server in der Anlage Zugang zu den Typenschildinformationen der Feldgeräte hat, also zu den statischen Feldgeräteparametern, wie beispielsweise Hersteller und Modelltyp, mit denen ein Feldgerät eindeutig identifiziert werden kann [6]. Dies kann durch Zugriff über HART, Profibus PA oder Foundation Fieldbus geschehen, über Remote-I/O-Komponenten oder Komponenten, die ebenfalls einen OPC-UA-Server besitzen. Der zentrale OPC-UA-Server, auch als NOA-Aggregated OPC-UAServer bezeichnet, enthält das NOA-Informationsmodell. In diesem sind die für diesen Use Case benötigten Typenschildinformationen in eindeutiger Semantik im Informationsadressraum hinterlegt. Die Eindeutigkeit wird dadurch erreicht, dass die Parameter in einem standardisierten Repository, dem IEC CDD Dictionary (nach IEC 61987), sowohl mit ihren Attributen, beispielsweise Datentyp, Name, Beschreibung, als auch mit einem weltweit eindeutigen Identifikator, der sogenannten IRDI, eingetragen sind.

Damit meldet die Anlage automatisch, wenn sich ihr As-built-Stand ändert, und die Anlageplanungsunterlagen können immer auf dem neuesten Stand gehalten werden - ohne Redlining, ohne Papier, ohne händische Übertragungen. Wartungs- und Umbauarbeiten werden dadurch erheblich erleichtert und übersichtlicher. Das Anlagenmodell im Engineeringwerkzeug bleibt keine Momentaufnahme.

\subsection{Aufbau des Demonstrators}

Quelle der Daten für den Use Case sind die in der Anlage verbauten Feldgeräte (Bilder 9 und 10). Die Verarbeitung der entsprechenden Parameter wird in einem 


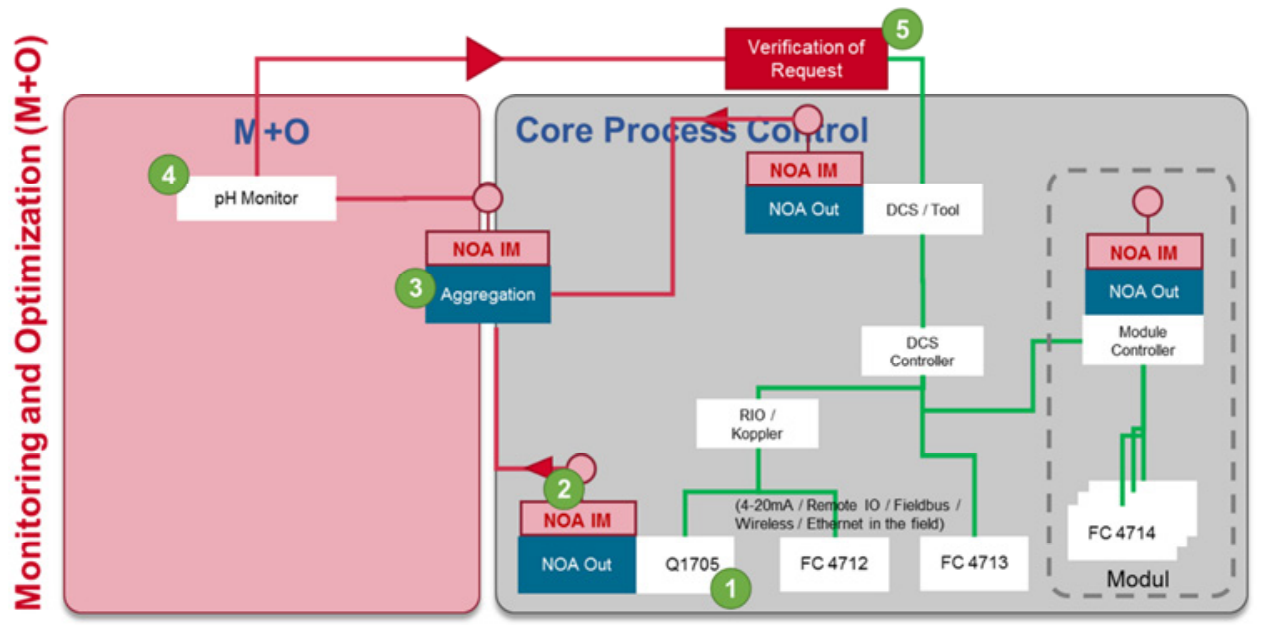

BILD 11: Architektur des NOA/PAT-Demonstrators

Engineeringwerkzeug vorgenommen, in dem sich die As-planned-Anlageplanungsunterlagen befinden. Das Engineeringwerkzeug greift über einen internetfähigen Zugang des NOA-Aggregated OPC-UA-Servers auf das As-built-Informationsmodel der Anlage zu. Zwischen den Feldgeräten und dem NOA-Aggregated OPC-UAServer muss eine Kommunikationsverbindung hergestellt werden. Diese kann bei Bestandsanlagen, wie zuvor beschrieben, vielfältig umgesetzt werden.

\subsection{Ergebnisse}

Für die Live-Demonstration wurde die IGR-Testanlage über ihren NOA-Aggregated OPC-UA-Server mit der Azure-Cloud von Aucotec verbunden. Das Werkzeug Engineering Base (EB) empfängt die Use-Casebezogenen Live-Daten der Demoanlage in definierten Intervallen (hier alle 2 s). Zuvor wurden in EB die As-planned-Anlageplanungsunterlagen in Form eines R\&I-Fließbildes und einer PLT-Stellenliste angelegt. In diesen EB-Anlageplanungsunterlagen erscheinen dann an jeder Stelle, die einen Messumformer oder Aktuator darstellt, die Hinweise zur Änderung. Durch diese Hinweise werden alle Änderungen dokumentiert und es können alle nötigen Anpassungen eindeutig und effektiv vorgenommen werden: zum Beispiel Verdrahtung anpassen, Spezifikationsblätter aktualisieren oder neue Revisionsstände erzeugen.

Anhand eines live vorgeführten Gerätetausches konnte die Änderungserkennung in EB visualisiert werden. Die Parameter gelangten in diesem Fall über Profibus PA und den bereits mit einem NOA-konformen OPC-UA-Server ausgestatteten Remote I/O in den NOAAggregated OPC-UA-Server.

\section{NOA USE CASE 3: ONLINE-ANALYSE VON PAT 5.1 Aufgabenstellung}

Der dritte Demonstrator beschäftigt sich mit der OnlineÜberwachung von Prozessanalysetechnik (PAT)-Systemen (TU Dresden/Yokogawa). PAT-Module bieten eine gute Möglichkeit, den Prozess in Bezug auf Qualität und Effizienz zu optimieren. Sie können jedoch relativ komplex und anfällig für Fehler unterschiedlichster Art sein. Daher ist eine kontinuierliche Kenntnis über den Gesundheitszustand des Systems notwendig, um die Zuverlässigkeit und die Qualitätsmerkmale des Systems abzuschätzen und dementsprechend die Regelung des Prozesses anzupassen.

Innerhalb der Prozessanalytik ist der pH-Wert einer der häufigsten und wichtigsten Qualitätsparameter. Obwohl das Messprinzip relativ einfach ist, gestaltet sich die effiziente Nutzung durch den prozessabhängigen Verschleiß und Verbrauch nicht trivial. Mögliche Fehlerursachen sind Defekte oder Beschädigungen des Sensors oder des Kabels, Verschmutzung, Abwesenheit von Medium, Vergiftung des Elektrolyts und Beeinflussung durch Druck und Temperatur. Diese Fehlerursachen und Probleme lassen sich durch die entsprechenden Instandhaltungsmaßnahmen (Kalibrierung, Reinigung, Reagenzien auffüllen, Austausch) entweder präventiv vermeiden oder man kann ihnen reaktiv begegnen. Die Häufigkeit des Fehlerauftritts und die notwendigen präventiven Instandhaltungsmaßnahmen sind aber stark anwendungsabhängig.

Der Demonstrator stellt eine typische Situation in heutigen Anlagen dar: Der pH-Wert wird für MPC (Model Predictive Control) im Leitsystem genutzt. Dazu wird der pH-Wert des Sensors mit 4-20 mA ausgelesen, wohingegen die Möglichkeit, Zusatzinformationen (beispielsweise die Referenzelektrodenimpedanz) über HART zu beziehen, nicht genutzt wird. Um einer Verschmutzung 


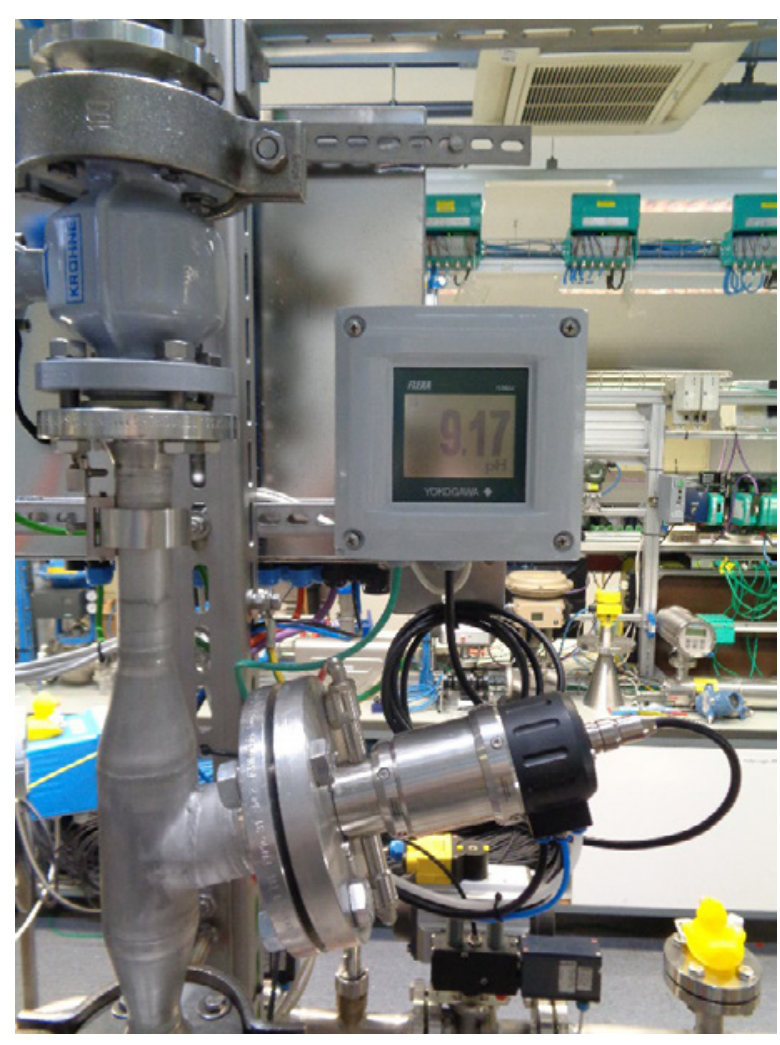

BILD 12: pH-Analyse-Gerät in der IGR-Anlage

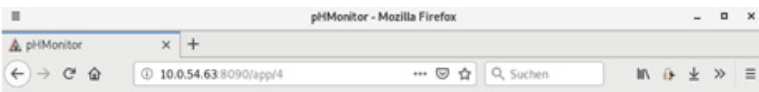

App IGR

Details

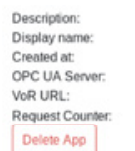

IGR tesplant 9 Hoeche

PHSensot
02112018130347

opc.tep: $/ / 1010.192 .1 .126: 4996$

nettp://10.6.54.63:8006/externa1/subast

Delete App

Analytics Information
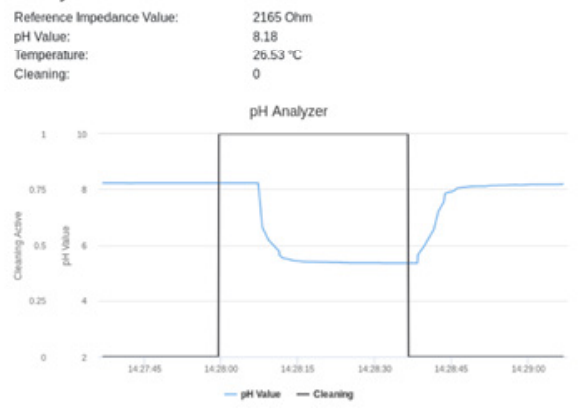

BILD 13: Screenshot des pH-Monitors während einer Reinigung des Sensors zu begegnen, wurde eine Wechselarmatur mit einer automatischen Reinigung installiert, die über das Prozessleitsystem (PLS) angesteuert wird. Die Aufgabe besteht nun in einer effizienten Überwachung der pH-Analyse.

\subsection{Aufbau des Demonstrators}

Der Demonstrator besteht aus fünf Komponenten (siehe Bild 11):

1| Ein Yokogawa FLXA 202 pH-Wert-Analysator mit einer automatischen Reinigung (genauer in Bild 12 dargestellt).

2 | Die Zusatzinformationen der pH-Sonde (Impedanzen, Temperatur, ...) werden über eine zusätzlich installierte NOA-Informationsdiode ausgelesen. Dies bewerkstelligt ein Raspberry Pi mit einem HART-Modem. Diese Zusatzinformationen werden über OPC UA bereitgestellt.

3| Ein read-only NOA-Aggregated OPC-UA-Server kombiniert diese Sensorinformationen mit einem Flag ReinigungAktiv aus dem PLS.

4. Die M+O App pH-Monitor (siehe Bild 13) kann aus diesen Informationen einen Elektrodenbruch (durch Anstieg der Impedanz), die Verschmutzung der Elektrode (Abfallen der Referenzimpedanz) und eine Sensoralterung und -verschmutzung (geändertes dynamisches Verhalten des $\mathrm{pH}$ Werts während der automatischen Reinigung) erkennen. Diese läuft als NodeJS-Anwendung auf einem Server an der TU Dresden.

5| Die App sendet bei Erkennen eines Fehlers einen Request an das PLS über die Verificationof-Request (VoR)-Komponente [7], sodass kein separates Dashboard für den Operator oder eine Benachrichtigung per E-Mail notwendig ist. Die VoR-Komponente läuft ebenfalls auf einem Server an der TU Dresden und stellt sicher, dass nur verifizierte Requests das PLS erreichen.

6| Das Leitsystem ist durch ein Centum VP realisiert, das den Request als Operator Message anzeigt.

\subsection{Ergebnisse}

Der Demonstrator ist in der Lage, das wirtschaftliche Potenzial einer NOA/PAT-Anwendung in den folgenden zwei Bereichen aufzeigen:

Kurzfristig: Kontext für einen optimalen MPC-Betrieb: Eine NOA/PAT-Anwendung kann Alterungseffekte und Fehler zuverlässig erkennen, welche die optimale Regelung durch den MPC verhindern. In dieser Weise kann der Operator kurzfristig benachrichtigt werden, wenn das PAT-System unzuverlässig läuft und der MPCController dementsprechend unsichere Entscheidungen trifft, die von ihm manuell zu beobachten sind (Operator Message PAT needs recalibration/cleaning/etc! Please monitor MPC closely). 
Langfristig: Optimierung der Reinigungszyklen: Als weiteren Vorteil kann die NOA/PAT-Anwendung präzise Kurvenverläufe und Historien für eine langfristige Optimierung der Instandhaltungsintervalle zur Verfügung stellen. So können Wartungskosten gespart werden.

\section{NOA USE CASE 4: DIMENSIONIERUNGSCHECK FÜR EINGEBAUTE FELDGERÄTE}

\subsection{Aufgabenstellung}

Der Demonstrator der TH Ingolstadt überprüft die Dimensionierung der PLT-Geräte der IGR-Testanlage. Hierbei kann es vorkommen, dass Geräte falsch dimensioniert wurden, etwa weil die Geräteauswahl aus einem Vorgängerprojekt ungeprüft übernommen wurde. In der Folge kann dies dazu führen, dass die Messung eines Sensors in einem ungünstigen Bereich erfolgt, wie es beispielsweise bei einem MID (magnetisch induktiven Durchflussmessgerät) bei sehr geringer Durchflussmenge der Fall ist. Als Beispiel für die Aktorik kann ein Regelventil angeführt werden, das die meiste Zeit nahe der Endlagen - und damit de facto als Auf-Zu-Ventil - betrieben wird.

Das NOA-Informationsmodell der Feldgeräte wird durch einen NOA-Aggregated OPC-UA-Server zur Verfügung gestellt (siehe Bild 14). Die Umsetzung des Use Cases entspricht dabei dem folgenden Ablauf:

- Zunächst wird ein Scan (OPC-UA-Browse) des Informationsmodells des NOA-Aggregated OPC-UA-Server durchgeführt.

- Für die gefundenen Geräteinstanzen werden die Identifikationsdaten gelesen.

- Anschließend werden für die im Informationsmodell enthaltenen Messbereichsgrenzen, die Einheit des Prozesswertes sowie den Prozesswert selbst Subscriptions erstellt, sodass Änderungen automatisch übertragen werden. Damit wird zum einen die Historie der Prozesswerte für die Auswertung aufgebaut. Zum anderen würde darüber eine gegebenenfalls vorgenommene Änderung der Prozesswerteinheit bekanntgegeben.

- Aus der Historie der Prozesswerte wird dann die Verteilung der Prozesswerte über den Messbereich berechnet sowie eine Kennzahl, die die Ausnutzung des Messbereichs repräsentiert.

\subsection{Aufbau des Demonstrators}

Der Demonstrator ist wie folgt aufgebaut: Ein Cloud Gateway verbindet sich als OPC-UA-Client mit dem NOAAggregated OPC-UA-Server und sendet die Gerätedaten über MQTT an die Cloud-Plattform 247FactoryNet (siehe Bild 15). Die Cloud-Plattform bildet die Geräte der Anlage als digitale Zwillinge ab [8]. Die digitalen Zwillinge enthalten zum einen die Daten des NOA-Informationsmodells. Zum anderen lassen sich diese mit beliebigen weiteren Daten anreichern oder mit anderen digitalen Zwillingen in Beziehung setzen. Das Cloud-Gateway führt, wie im Ablauf beschrieben, einen Scan des

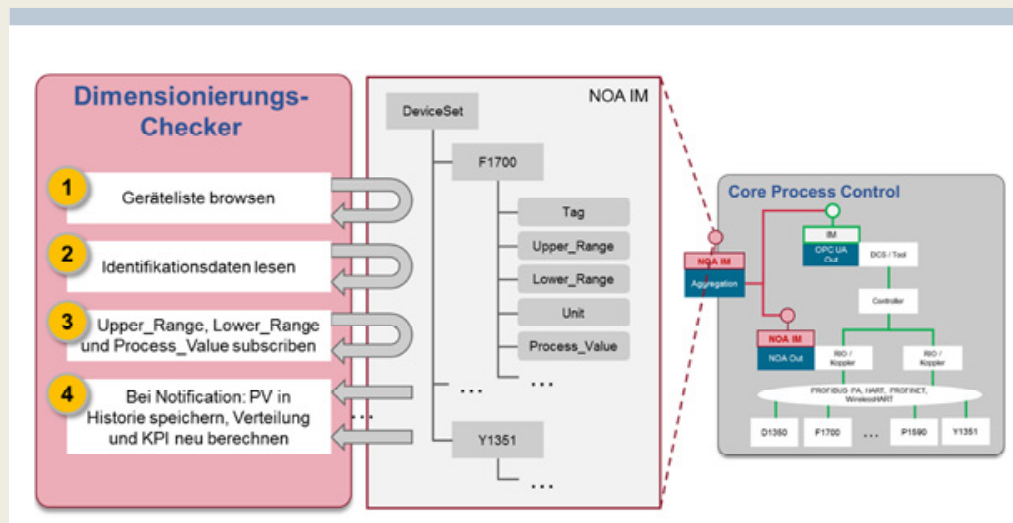

BILD 14: Prinzipieller Ablauf

Informationsmodells des NOA-Aggregated OPC-UAServer durch und liest die Identifikationsdaten der Feldgeräte. Anschließend erfolgt ein Abgleich mit den in der Cloud-Plattform bereits gespeicherten digitalen Zwillingen. Für neue Geräteinstanzen werden neue digitale Zwillinge erzeugt. Bei Wertänderungen im NOAAggregated OPC-UA-Server werden automatisch Updates übertragen. Damit wird die Historie der Prozesswerte in den digitalen Zwillingen aufgebaut. Aus der Historie der Prozesswerte wird zum einen laufend ein Histogramm aktualisiert, das die Verteilung der Prozesswerte über den Messbereich darstellt. Zum anderen wird ein Kennwert berechnet, der die Ausnutzung des Messbereichs repräsentiert. Die Kennwerte werden sortiert in einer Liste dargestellt, sodass sich ein unmittelbarer Überblick über die Feldgeräte der Anlage gewinnen lässt. Bei Bedarf können die detaillierte Darstellung der Prozesswerthistorie sowie der aktuelle Live-Prozesswert aufgerufen werden.

\subsection{Ergebnisse}

In der IGR-Testanlage wurde unter anderem erfolgreich ein absichtlich überdimensionierter MID-Durchflussmesser identifiziert: Das Feldgerät ist mit einem Sensor mit einem Messbereich von 0-20000 l/h ausgestattet, wird aber in der Regel lediglich mit $700 \mathrm{l} / \mathrm{h}$ betrieben. Darüber hinaus ließen sich typische Betriebspunkte der Testanlage identifizieren. Mit dem Demonstrator konnte der Use Case somit erfolgreich umgesetzt werden.

\section{NOA: STATUS UND AUSBLICK}

Die vier in diesem Artikel beschriebenen Use Cases, zeigen den Hauptfokus für die Namur Open Architecture. Es geht bei NOA nicht um eine technische Revolution oder Weiterentwicklung der Kernautomatisierungssysteme, sondern um eine vorwiegend durch Monitoring + Optimization Use Cases getriebene Entwicklung. Dies erfüllt die in Europa dominierende Anforderung, beste- 


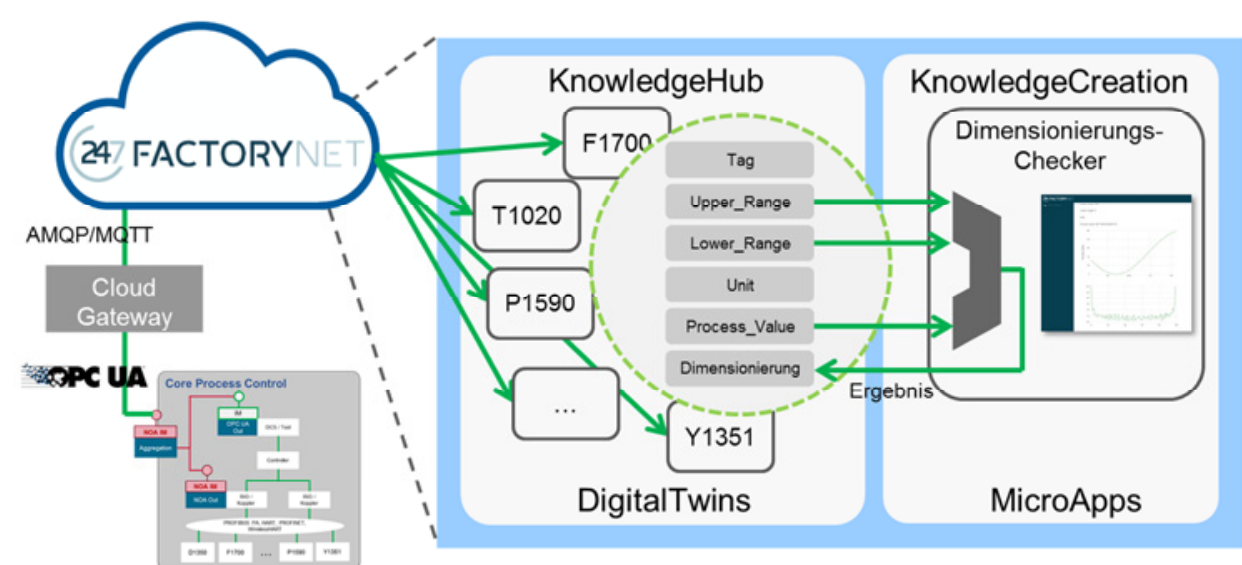

BILD 15: Aufbau des Demonstrators

hende (Brownfield-) Produktionsanlagen in die neue digitale Welt der Industrie $4.0 \mathrm{zu}$ bringen.

Zusammen mit den für die Namur-Hauptsitzung 2018 entwickelten Demonstratoren zeigt die Testanlage der IGR bei Bilfinger Maintenance $\mathrm{GmbH}$, was aktuell mit kommerziell verfügbaren Lösungen bereits umgesetzt werden kann. Es sind aber auch die immer noch

\section{REFERENZEN}

[1] Klettner, C., Tauchnitz, T., Epple, U., Nothdurft, L., Diedrich, C., Schröder, T., Grossmann, D., Banerjee, S., Krauss, M., latrou, C., Urbas, L. (2017). Namur Open Architecture. atp magazin 59(01-02), 20-37.

[2] Klettner, C., Krauss, M. (2017): Namur Open Architecture: Die Namur-Pyramide wird geöffnet für Industrie 4.0. atp plus 2017(01-02), 28-33.

[3] Tauchnitz, T. (2018): NAMUR Open Architecture - Auf dem Weg zu Industrie 4.0. atp plus 2018(01), 6-15.

[4] NAMUR. (2013). NE 148: Anforderungen an die Automatisierungstechnik durch die Modularisierung verfahrenstechnsicher Anlagen. NAMUR: www.namur.net

[5] Schröder, T., Diedrich, C. (2018). NOA schafft Voraussetzungen für neue digitale Dienste. atp magazin 60(01-02), 48-51.

[6] Nothdurft, L., Epple, U. (2018). Plug-and-ProduceProzess mit NOA automatisieren. atp magazin 60(01-02), 44-46.

[7] latrou, C.P., Graube, M., Urbas, L, Henrichs, T.-P., Erben, S. (2018): NOA Verification of Request. atp magazin 60(01-02), 60-69.

[8] Grossmann, D., Banerjee, S., Schmied, S., (2018): Feldgerätedaten mit NOA in Cloud-Applikationen nutzen. atp magazin 60(01-02), 52-58. bestehenden Lücken für eine flachendeckende Umsetzung des NOA-Konzepts zu erkennen: Die aktuellen technischen Lösungen skalieren noch nicht ausreichend und fordern immer noch einen hohen individuellen Konfigurationsaufwand, um die Daten zu erfassen, welche die Use Cases ermöglichen. Auch für die Konzepte und Lösungen zur Sicherstellung von Automation Security, die für die verschiedensten Datenflüsse zwischen dem Core-ProcessControl-Bereich und dem $\mathrm{M}+\mathrm{O}-$ Bereich definiert werden müssen, gibt es heute noch zu wenig praktische und skalierbare Lösungen und Best Practices, die der Anwender als Leitfaden benutzen kann.

Das Schließen dieser Lücken ist das Ziel für NOA im Jahr 2019. Die Namur und der ZVEI (Zentralverband Elektrotechnik- und Elektronikindustrie e.V.) haben dafür ein gemeinsames Projekt aufgesetzt, in dem die funktionalen Anforderungen von den Anwendern gemeinsam mit den Herstellern in technischen Spezifikationen und Best Practices umgesetzt werden sollen. Die drei Schwerpunkte der Zusammenarbeit sind genau die drei NOA-Bausteine, die in diesem Beitrag vorgestellt wurden:

- ein einheitliches NOA-Informationsmodell,

- die NOA-Diode und

- die Verification-of-Request-Lösung.

Für die letzten beiden Themen müssen hauptsächlich die Automation-Security-Anforderungen berücksichtigt werden. Für das Thema NOA-Informationsmodell ist auch die Abstimmung mit anderen Organisationen wie der FieldComm Group, der Profibus-Nutzerorganisation und eben auch der OPC Foundation erforderlich, sodass das Informationsmodell breit in der Industrie getragen wird.

Wenn diese Elemente spezifiziert sind, können sie in marktgängigen Produkten realisiert werden. 2019 wird also ein entscheidendes Jahr sein für NOA: Es soll der Schritt gemacht werden von einem vielversprechenden Konzept zum Status reif für den breiten Einsatz. 


\section{AUTOREN}

Dr.-Ing. JAN DE CAIGNY (geb. 1982) promovierte an der Katholieke Universiteit Leuven im Bereich Regelungstechnik. Bei BASF war er von 2011 bis 2016 tätig bei der PLT-Planung von Investitionsprojekten in Belgien und Brasilien. Seit 2018 leitet er ein Fachgebiet für industrial Internet of Things und OT-Datenkonnektivität. In der NAMUR leitet er den Arbeitskreis 2.8 Automatisierungsarchitekturen.

\section{BASF SE,}

Carl-Bosch-Strasse 38

67056 Ludwigshafen am Rhein,

Tel. +49 (0) 6216049396 ,

E-Mail: jan.de-caigny@basf.com

Dr.-Ing. THOMAS TAUCHNITZ (geb. 1957) studierte in Hannover Elektrotechnik und promovierte im Bereich der Regelungstechnik. Nach 32 Berufsjahren im Industriepark Frankfurt-Höchst und 25 Jahren NAMUR-Engagement ist er seit 2018 selbständiger Consultant im Bereich Industrie 4.0, Automatisierungstechnik und Engineering-Tools. Er ist Mitglied des NAMUR-AK 2.8 und Chefredakteur „Industry“ des atp magazin.

RONNY BECKER (geb. 1978) ist Laboringenieur im Geräteprüflabor der Bilfinger Maintenance im Industriepark Höchst. Seine Tätigkeitschwerpunkte dort sind die Kommunikation zwischen Automatisierungskomponenten in der Prozesstechnik sowie Gerätetests im Rahmen von Typprüfungen. Er ist verantwortlich für die Errichtung und den Betrieb der IGR-Test- und Demoanlage.

Prof. Dr.-Ing. CHRISTIAN DIEDRICH (geb. 1957) hat technische Kybernetik und Automatisierungstechnik studiert und lehrt und forscht an der Otto-vonGuericke-Universität und am Institut für Automation und Kommunikation (ifak) e.V. in Magdeburg. Seine Schwerpunkte liegen auf dem Gebiet der Automation in digital betriebenen Produktionssystemen basierend auf informations- und wissensbasierten Methoden. Dazu gehören vor allem die industrielle Kommunikation, Integrations- sowie Beschreibungsmethoden. Er leitet unter anderem den Gemeinschaftsarbeitskreis „Semantik und Interaktion von I4.0-Komponenten“ der GMA/Plattform Industrie 4.0 und ist Convenor in der IEC-Strandardisierung von EDDL, FDT und FDI.

M. Sc. TIZIAN SCHRÖDER (geb. 1991) ist Mitarbeiter am Lehrstuhl Integrierte Automation an der Ottovon-Guericke-Universität Magdeburg. Zuvor stu- dierte er Wirtschaftsingenieurwesen für Elektro- und Informationstechnik mit dem Schwerpunkt Automatisierungstechnik. Seine Hauptarbeitsfelder sind semantische Beschreibungsmethoden als Grundlage für Interoperabilität in der digitalen Fabrik sowie Interaktionsmodelle für I4.0-Komponenten.

Prof. Dr.-Ing. DANIEL GROSSMANN (geb. 1977) studierte an der TU München Maschinenbau mit den Schwerpunkten Informationstechnik und Automatisierungstechnik. Nach seiner Promotion im Bereich Geräteintegration, für die er 2008 den Namur-Award erhielt, wechselte er in die Konzernforschung der ABB AG. Seit 2013 ist er Professor für Ingenieurinformatik und Datenverarbeitung an der Technischen Hochschule Ingolstadt. Zu seinen Forschungsthemen zählen industrielle Kommunikation, Geräteintegration und Informationsmodellierung in der Automatisierungstechnik als Basis für Industrie 4.0, Cyber Physical Systems und Industrial Internet of Things.

M.Sc. SUPRATEEK BANERJEE (geb. 1986) ist wissenschaftlicher Mitarbeiter an der TH Ingolstadt. Er hat seinen Masterabschluss an der Universität Stuttgart im Studiengang Embedded Systems erworben. Sein Hauptarbeitsgebiet ist die Aggregation von Informationsmodellen in der Fertigungs- und Prozessindustrie.

Prof. Dr.-Ing. LEON URBAS (geb. 1965) leitet an der TU Dresden die Professur für Prozessleittechnik und die Arbeitsgruppe Systemverfahrenstechnik. Er ist Mitglied des Vorstands des Process-to-OrderLab und Sprecher des DFG-Graduiertenkollegs CDCPPS. Forschungsfelder sind offene Informationsund Automationsarchitekturen (MTP, NOA, OPAF, OPC UA, ...) und Mensch-Technik-Kollaboration in der Prozessindustrie.

Dr.-Ing. MARKUS GRAUBE (geb. 1985) hat nach seinem Mechatronik-Studium in Dresden zum Thema Informationsräume für industrielle Daten promoviert. Nun ist er Geschäftsführer des Process-toOrder-Lab an der TU Dresden, in dem interdisziplinär die Möglichkeiten und Auswirkungen der Digitalisierung in der Prozessindustrie zum Beispiel durch NOA und MTP untersucht und an realen Anlagen evaluiert werden. 